\title{
Feasibility of Using Infant Testing during Immunization to Estimate Mother-to-Child- Transmission Rates in Zambia
}

Joseph Simbaya ( $\mathbf{j}$ j.simbaya@unza.zm )

https://orcid.org/0000-0001-7518-6370

\section{Patricia Funjika}

University of Zambia, Institute of Economic and Social Research

\section{Arthur Moonga}

University of Zambia Institute of Economic and Social Research

John Mwale

National AIDS Council

Chipepo Kankasa

University Teaching Hospital, Pediatric HIV Centre of Excellence

Research article

Keywords: HIV, Mother-to-child transmission, Africa, Children

Posted Date: March 16th, 2020

DOI: https://doi.org/10.21203/rs.3.rs-17305/v1

License: (c) (1) This work is licensed under a Creative Commons Attribution 4.0 International License. Read Full License 


\title{
RESEARCH
}

\section{Feasibility of Using Infant Testing during Immunization to Estimate Mother-to-Child-Transmission Rates in Zambia}

\author{
Joseph Simbaya ${ }^{1 *}$, Patricia Funjika ${ }^{1}$, Arthur Moonga ${ }^{2}$, John Mwale ${ }^{3}$ and Chipepo Kankasa ${ }^{4}$
}

\footnotetext{
${ }^{*}$ Correspondence:

j.simbaya@unza.zm

${ }^{1}$ Institute of Economic and Social Research (University of Zambia) Munali Road, 10101 Lusaka, Zambia

Full list of author information is available at the end of the article
}

\begin{abstract}
Background: This study piloted the feasibility of infant testing in immunization services as a strategy for estimating MTCT rates among the population of HIV exposed infants at national and subnational levels in Zambia.

Methods: The study recruited a cross-sectional nationally representative sample of 8,042 caregiver-baby pairs in 38 high volume immunization sites in 7 towns across 3 provinces of Zambia. All mothers who brought their children below the age of one year for immunization at the study facilities were invited to participate in the study. All consenting mothers were interviewed and blood drawn from their babies for; rapid HIV antibody test to determine exposure and DNA PCR test for samples of all HIV-exposed babies to determine HIV infection.
\end{abstract}

Results: Of 8,042 recruited caregiver-baby pairs, 1,407 (17.5\%) babies were HIV-exposed. More than 90 percent of all mothers of HIV exposed infants reported that they attended ANC visits more than two times and facility based deliveries stood at 92 percent. Exclusive breastfeeding among HIV exposed infants reduced with increase in age of infant; it was highest at 6 weeks $(82 \%)$ followed by 10 weeks (74\%), 14 weeks ( $74 \%)$ and 9 months (4\%). MTCT rates were relatively lower than what was reported before in subnational studies and stood at 4.94 percent among Penta 1 seekers, 3.08 percent among Penta 2 seekers, 2.63 percent among Penta 3 seekers and 5.15 percent among Measles vaccination seekers. The overall MTCT rate stood at 4.05 percent. About 46 percent of HIV positive babies were male compared to 54 percent females. Babies of mothers below the age of 25 years accounted almost half (47\%) of all HIV infected babies in the study. Reported exclusive breastfeeding among HIV positive babies was 79 percent for Penta 1 seekers, 78 percent for Penta 2 seekers and 100 percent for Penta 3 seekers.

Conclusion: The study succeeded in estimating the MTCT rates using infant testing in immunization services, thereby demonstrating that it is feasible to use routine infant testing in immunization services as a strategy for estimating MTCT rates among the population of HIV-exposed infants in Zambia.

Keywords: HIV; Mother-to-child transmission; Africa; Children

\section{Background}

Vertical transmission of Human Immunodeficiency Virus (HIV) is the most common source of paediatric HIV infection in Zambia [1]. In 2016, around 8,900 children became newly infected with HIV in Zambia indicating a 56 percent decline from 20,000 new paediatric infections in 2009 [2, 3]. Mother-to-Child Transmission of HIV 
(MTCT) ranges from 15 percent to 45 percent in the absence of any intervention. MTCT can however be reduced to below 5 percent with effective interventions during pregnancy, labour, delivery and breastfeeding. Intervention include inter alia; Antiretroviral Treatment (ART) for the HIV positive pregnant woman or mother and a short course of antiretroviral drugs for the exposed infant, facility based delivery, and appropriate breastfeeding practice $[4,5]$.

Zambia first introduced its rigorous Prevention of Mother-to-Child Transmission of HIV (PMTCT) programme in 1999 and has been committed to virtual elimination of MTCT since 2010 [6]. Zambia's prevention and treatment of HIV infection guidelines requires the testing of all women receiving Antenatal Care (ANC) and enrolment of all those that are found positive into HIV care and treatment [7].

Despite Zambia's continued efforts to eliminate MTCT, measuring of MTCT at national level remains a challenge despite the routine documentation of infant testing data through the PMTCT programme. Previous studies on efficacy of routine HIV testing data from PMTCT programmes have indicated that the data is largely unavailable and when it is available, it is usually incomplete, inaccurate and not available on time $[8,9,10,11,12]$, thus partly explaining the reluctance to use it for estimating MTCT rates. Previous efforts to measure MTCT rates have therefore primarily focused on subnational levels.

Measurement of MTCT is critical in planning and meeting the needs of paediatric HIV prevention, care, and treatment services for children, as well as evaluating the effectiveness of PMTCT interventions [13, 14]. The Government of the Republic of Zambia and the international community are cognizant of the challenge in measuring MTCT. This study was therefore conducted with the aim of assessing the feasibility of infant testing in immunization services as a strategy for estimating MTCT rates among the population of HIV exposed infants (HEI).

The Zambia Programme on Immunization seeks to immunize infants against:

- Tuberculosis with the Bacille Calmette Guerin (BCG) vaccine at birth;

- Polio with oral polio vaccine (OPV) at 0 (birth), 6, 10 and 14 weeks and inactive polio vaccine (IPV) at 14 weeks;

- Diphtheria, Pertussis, Tetanus, Haemophilus Influenza type B, Hepatitis B with three doses of the Penta vaccine at 6,10 and 14 weeks of age (DPTHibHep1, DPTHibHep2 and DPTHibHep3 respectively);

- Diarrhoea with Rota vaccine at 6 and 10 weeks;

- Streptococcal Pneumonia with the pneumococcal conjugate vaccine (PCV) at 6, 10 and 14 weeks

- Measles and Rubella vaccines at 9 and 18 months of age;

HIV testing for HEI in Zambia is aligned to immunization and is done at birth ( during BCG and OPV), 6 weeks (during OPV, DPT1 and Rota), 14 weeks (during OPV, IPV, and PCV), 9 months (during Measles and Rubella), 12 months, 18 months (during measles and rubella), and 24 months [7]. This study was guided by immunization service statistics which suggest that client flow is fairly evenly divided among the three Penta vaccines and Measles vaccine, with the highest proportion at DPTHibHep1 and the lowest at measles. 


\section{Methods}

This was a variant of sentinel surveillance study which followed a cross-sectional approach. The study took advantage of Zambia's highly successful childhood immunization program which starts at birth (BCG) through to 18 months (measles and rubella). Using these services, the study recruited mothers and infants for HIV testing to provide data for measuring the MTCT rates. The study sought to obtain blood samples from 8,000 infants under the age of 12 months distributed across 38 health facilities from across three provinces (Copperbelt, Lusaka and Southern provinces of Zambia). It was estimated that this would yield a nationally representative sample of approximately 1,240 HEI.

The nationally representative sample of 1,240 was determined using an average HIV prevalence among women 15-49 of $21.6 \%$ in 2007 [15] and adjusting for lower prevalence among pregnant women than non-pregnant women (11.6\% vs. $16.6 \%$ nationally) and the pregnancy rate ( $8 \%$ currently pregnant), it was estimated that $15.5 \%$ of women with a recent birth are HIV-positive. Assuming no differential mortality, equal likelihood of seeking vaccination and equal refusal rates, 1,240 (15.5\%) out of 8,000 infants tested would be HIV-exposed. The precision of the estimated age-specific MTCT rates would depend on the age distribution of HIVexposed infants in the sample.

In light of national vaccination norms promoting Penta vaccine booster shots (Penta-2 and Penta-3) at 4-week intervals, data collection at any one vaccination site was limited to a 4-week period to avoid re-peat testing of infants. Participants were concurrently recruited at all vaccination sites in the study until a total sample of 8,000 consenting participants was achieved.

During the data collection period, all consenting caregivers of infants between 0 and 12 months of age who were receiving vaccinations in study sites were recruited. Inclusion criteria for care-givers were: Caregiver of age 16 and over, attending vaccination for DPT1, DPT2, DPT3 or measles with infants younger than 12 months of age; and physically and mentally capable of providing informed consent for the interview.

This study involved interviewing the caregivers and taking blood samples from participating infants and testing them for both HIV antibodies (all recruited infants) and HIV DNA (infants with positive antibody tests). As part of obtaining informed consent, all caregivers were informed about the need for and difference between the two tests and that the presence of HIV antibodies in the baby's blood meant that the mother was HIV-positive. However, anti-body test results were only availed to the caregivers on request and upon receiving adequate counselling. For quality assurance and infant safety purposes, the study used certified HIV counsellors and Nurses working at the respective health facility with experience in DBS collection. All study counsellors and nurses received additional training from the research team to ensure that they had the knowledge and skills to educate mothers about the testing procedures and to properly take and preserve infant blood samples. To avoid irritating the child, only one sample was drawn from which an antibody test was done to determine exposure as well as DBS to determine actual HIV infection.

The MTCT rate was calculated as a proportion of infant blood samples with a positive PCR test result among infants with a positive HIV antibody test result 
or with known HIV-positive mothers; the numerator was the number of all PCR positive babies and the denominator was the number of all HIV exposed infants.

\section{Results}

Study Population

The population for the study encompassed all caregiver-infant pairs attending immunisation for Penta vaccine 1, to 3 , and measles vaccine for 9 months at the study sites. Of the 8,522 caregiver-infant pairs that were approached, 8,289 pairs consented for interviews and 8,042 consented for infant HIV testing representing a 97.27 percent and 94.37 percent response rates for interviews and infant testing respectively as shown in Table 1. Copperbelt province had the highest interview response rate (98.59\%) followed by Southern $(97.04 \%)$ and Lusaka (96\%) provinces and there was an almost equal distribution of infant testing response across provinces.

Table 1 further shows that Southern province recruited the highest number of participants $(2,960)$ followed by Copperbelt $(2,713)$ and Lusaka $(2,369)$. A total of seven districts participated in the study; Ndola, Kitwe and Chingola from Copperbelt Province, Livingstone, Choma, and Mazabuka from Southern Province, and Lusaka from Lusaka Province.

\section{TABLE 1 HERE}

\section{Social demographics}

Male babies accounted for about 52 percent of the sample while female babies accounted for about 48 percent (Figure 1). Overall, slightly more babies recruited for the study came to the facility to receive Penta 1 (27\%). The rest came for Penta $2(23 \%)$, Penta $3(25 \%)$ and Measles $(26 \%)$.

FIGURE 1 HERE

The majority $(47 \%)$ of the mothers were aged less than 25 years old. Similarly, the majority were married ( $82 \%)$, had secondary education $(62 \%)$ and were not employed (67\%) (Table 2).

\section{TABLE 2 HERE}

\section{HIV Infant Exposure}

The HEI accounted for 17.5 percent of the overall infants tested for HIV antibody. Slightly more male infants (10\%) were HIV exposed than females (8\%). Of all HEI $(\mathrm{N}=1,409)$, more male babies $(56 \%)$ were exposed than female babies $(44 \%)$. Slightly below half $(45 \%)$ of all HEI had mothers above 30 years old while about a quarter $(26 \%)$ had mothers in the age range of 25 to 29 years (Table 4 ).

There were more HEI who came for Penta 1 (27.7 \%) and Measles $(27.5 \%)$ vaccinations than those who came for Penta $2(20.7 \%)$ or Penta $3(24.1 \%)$. Overall, about 92 percent of all exposed infants were delivered in a health facility. Almost all mothers of exposed babies had tested for HIV (99 \%) and had attended three or more ANC visits $(90 \%$ ) (Table 4). Figure 2 presents the number of HII at national and provincial levels by immunization stage/type the baby came to receive at the time of drawing the sample. A total of $19 \mathrm{HIV}$ positive results were detected in infants who came for Penta 1. Penta 2 and Penta 3 each had 9 babies testing positive to PCR test while Measles had 20.

FIGURE 2 HERE 


\section{MTCT Rates in immunization services}

Of all exposed infants $(\mathrm{N}=1,409)$, more male babies ( 56 percent) were exposed than female babies Of the 1,409 exposed infants, 1,389 samples were successfully tested for HIV using DNA PCR. A total of 12 samples were rejected and 4 had missing results at the time of study completion. A total of 52 samples were reactive to the PCR test representing an overall rate of transmission of 3.75 percent for infants aged 6 weeks to 9 months. The MTCT rates at 6 weeks, 10 weeks, 14 weeks and 9 months were 4.7 percent, 2.8 percent, 2.1 percent and 5.0 percent respectively (Table 3).

\section{TABLE 3 HERE}

The highest proportion of HII was among infants who came to seek Measles vaccination $(36.5 \%)$ at 9 months followed by those who came to seek Penta $1(27.7 \%)$ at 6 weeks. Penta 2 Penta 3 accounted for 15 percent and 14 percent of HII respectively. There were more female HII $(51.9 \%)$ than male HII $(48 \%)$. Slightly over half $(51.9 \%)$ of all HII were among infants whose mothers were less than 25 years old. The majority $(80.1 \%)$ HII were delivered in health facilities while about 17 percent were delivered at home. The overall number of ANC attendance visits among mothers of HII was high (90.4\% had at least 3 ANC visits) and so was reported exclusive breastfeeding (51.9\%).

\section{TABLE 4 HERE}

\section{Discussion}

The study tested the feasibility of infant testing in immunization settings as a technique to determine MTCT rates among infants. The findings indicate that it is feasible, provided adequate resources for supplies (HIV test kids and reagents) are made available to cater for massive tests of all infants in immunization clinics. This will take a strain on existing supplies and will need adequate planning to ensure that all infants in immunization clinics are tested for exposure and all exposed infants are tested for HIV using DNA PCR. The success of the program, as indicated by previous studies will also depend on a strong data capture and documentation system, and robust routine data quality assessment (RDQA) [8, 10, 16, 17, 18].

Our study is the first to test the feasibility of this approach to determine MTCT rates in Zambia. However, this method is recommended by the World Health Organisation[19] and has been used and proven to be feasible in Malawi[20] and KwaZulu Natal in South Africa [21]. Our study makes a significant contribution to this growing body of knowledge. The study recruited an even distribution of babies across the four immunization types (Penta 1, Penta 2, Penta 3 and Measles) below the age of one year.

The transmission rate at 6 weeks stood at 4.7 percent and was consistent with the 2017 Joint United Nations Programme on HIV/AIDS (UNAIDS) estimate of 5 percent. The MTCT rate between 6 weeks and 9 months stood at about 3.75 percent, lower than the reported rates in earlier studies1 Copperbelt (4.37 percent) and Lusaka (4.52) provinces had the highest overall proportion of HIV exposed babies who tested positive to HIV. This is in line with the national HIV prevalence trends which are high in Lusaka (15.7\%) and Copperbelt provinces (13.8 \%) [14].

As expected, the highest rate of vertical transmission was detected among infants who came for Measles vaccination $(5.0 \%)$ This was followed by those who came for 
Penta 1 (4.7\%). It is likely that babies continue to become infected at all stages of immunization. This implies that the risk of infection is continuous and interventions are required throughout the PMTCT cascade until breastfeeding cessation. The majority of infants who tested PCR positive are of mothers below the age of 25 years. This is suggestive of the treatment naive mothers who either recently became aware of their status or are struggling to settle in their new sero-status. The study was successful in estimating the MTCT rates for infants below the age of 12 months thus indicating that infant testing in immunization services is a feasible strategy for estimating MTCT rates among the population of HIV exposed infants. Success of this strategy however requires mobilisation of resources and focusing them on inter alia; timely procurement and delivery of infant testing supplies, training on infant testing, timely testing of DBS and communication of results, establishment of robust RDQA to ensure that infant testing data is available on time, complete, accurate, reliable and is of high precision and integrity[22], and improving follow of mother-baby pairs[23].

\section{Conclusion}

This study suggests that it feasible to use immunization clinics to monitor both the maternal and infant HIV infections rates and that routine testing in immunization clinics can be used to identify exposed and infected infants early and link them to treatment. Follow-up mechanisms however, need to be put in place to ensure that mother-baby pairs who are not enrolled in the HIV treatment program are tracked and linked to treatment and care. There is further need for routine data quality assessment of PMTCT programme data in all facilities providing PMTCT services. Although this will require increased resources to implement, the long-term cost of estimating MTCT rates and evaluating the effectiveness of the PMTCT program will be reduced.

\section{Abbreviations}

ANC: Antenatal Care

ART: Antiretroviral treatment

BCG: Bacille Calmette Guerin

DBS: Dried Blood Spot

DNA: Deoxyribonucleic Acid

DPTHibHep: Diphtheria, Pertussis, Tetanus, Haemophilus Influenza type B, Hepatitis B

HEI: HIV Exposed Infant

HII: HIV Infected Infant

HIV: Human Immunodeficiency Virus

IPV: inactive Polio Vaccine

MTCT: Mother-to-Child Transmission

OPV: Oral Polio Vaccine

PCR: Polymerase Chain Reaction

PCV: Pneumococcal Conjugate Vaccine

PMTCT: Prevention of Mother-to-Child Transmission

RDQA: Routine Data Quality Assessment Joint United Nations Programme on HIV/AIDS (UNAIDS) 
Declarations

Ethics approval and consent to participate

Ethical approval for the study was sought from the University of Zambia Biomedical Research Ethics Committee (UNZABREC), a local institutional review board (IRB). Additional approvals were obtained from National Health Research Authority and the Zambian Ministry of Health at provincial, district and health facility levels. Only children whose biological parents consented were included in the study. Informed written consent was obtained from the biological parents of the infants before the interview. Additional written consent was obtained from the biological parents to draw blood from their infant.

\section{Consent for publication}

Not applicable

\section{Competing interests}

The authors declare that they have no competing interests.

Funding

The study was supported with funding from Centers for Disease Control and Prevention (CDC). The funding covered data collection, analysis and report writing.

\section{Availability of data and materials}

The datasets used and/or analysed during the current study are available from the corresponding author on reasonable request.

\section{Author's contributions}

JS led on study design, data collection, participated in the data analysis and interpretation of results, prepared the first draft and participated in later drafts of the article. PF participated in data analysis and interpretation and drafting of the article. AM participated in data collection, data analysis, interpretation and drafting of the article. JM participated in the study design, training of data collectors and drafting of the article. CK participated in the study design and review of the article. All authors have seen and approved the final version.

\section{Acknowledgements}

The authors would like to thank all the respondents, health facility staff and District Health Management Teams in Lusaka, Copperbelt and Southern Provinces who participated in the study. The authors would also like to thank the Zambian Ministry of Health and the main study funder, CDC. None of the funding organisations were involved in the analysis/ interpretation of data or the writing of this manuscript.

\section{Author details}

${ }^{1}$ Institute of Economic and Social Research (University of Zambia), Munali Road, 10101 Lusaka, Zambia. ${ }^{2}$ Institute of Development and Research, Mosi-oa-Tunya Road, 10101 Lusaka, Zambia. ${ }^{3}$ Zambia National HIV/AIDS/TB Council, Independence Avenue, 10101 Lusaka, Zambia. ${ }^{4}$ University Teaching Hospital HIV AIDS Program (UTH-HAP) , Burma Road, 10101 Lusaka, Zambia

\section{References}

1. Torpey, K., Mandala, J., Kasonde, P., Bryan-Mofya, G., Bweupe, M., Mukundu, J., Zimba, C., Mwale, C., Lumano, H., Welsh, M.: Analysis of hiv early infant diagnosis data to estimate rates of perinatal hiv transmission in zambia. PloS one 7(8) (2012)

2. UNAIDS: Start Free Stay Free AIDS Free 2017 Progress Report. Technical Report UNAIDS/JC2923E, UNAIDS (2017)

3. UNICEF: Evaluation of the UNICEF PMTCT / Paediatric HIV Care and Treatment Programme. Technical report, UNICEF (2017). https://www.unicef.org/evaldatabase/index_95015.html

4. WHO: HIV/AIDS: Mother-to-Child Transmission of HIV. Technical report, WHO (2019) https://www.who.int/hiv/topics/mtct/about/en/

5. Ndubani, P., Simbaya, J., Walsh, A., Dicker, P., Kamwanga, J., Brugha, R.: Tracking global hiv/aids initiatives and their impact on the health system in zambia. Lusaka: University of Zambia/Royal College of Surgeons in Ireland (2009)

6. A., A., Waldron, J.: Incremental Cost of Providing Key Services to Prevent Mother-to-Child Transmission (PMTCT) of HIV in Zambia. Technical report, Futures Group, USAID Health Policy Initiative, Costing Task Order (2013)

7. Ministry of Health: Zambia Consolidated Guidelines for Treatment and Prevention of HIV Infection. Technical report, Ministry of Health (MOH) [Zambia] (2018)

8. Mlambo, M.G., Peltzer, K., Koivu, A.: Prevention of mother-to-child transmission of hiv data completeness and accuracy assessment in health facilities of the nkangala district. Health SA Gesondheid (Online) 19(1), 1-8 (2014)

9. INESOR: Data Quality Assessment (DQA) Study Report for Development of the Impact Study Protocal on Prevention of Mother to Child Transmission (PMTCT) in Zambia. Technical report, INESOR/ University of Zambia (2013)

10. Moonga, A.M.: Prevention of Mother-to-Child Transmission of HIV Data Quality Assessment in Selected Health Facilities of the Southern Region of Zambia. (2017). https://www. semanticscholar.org/paper/Prevention-of-Mother-to-Child-Transmission-of-HIV: -Moonga/10802d4497a3c99d8a8342bed8110469185cb72a

11. Sirengo, M., Rutherford, G.W., Otieno-Nyunya, B., Kellogg, T.A., Kimanga, D., Muraguri, N., Umuro, M., Mirjahangir, J., Stein, E., Ndisha, M., et al.: Evaluation of kenya's readiness to transition from sentinel surveillance to routine hiv testing for antenatal clinic-based hiv surveillance. BMC infectious diseases 16(1), 113 (2016) 


\section{Figures}

12. Young, P.W., Mahomed, M., Horth, R.Z., Shiraishi, R.W., Jani, I.V.: Routine data from prevention of mother-to-child transmission (pmtct) hiv testing not yet ready for hiv surveillance in mozambique: a retrospective analysis of matched test results. BMC infectious diseases 13(1), 96 (2013)

13. Nguefack, H.L.N., Gwet, H., Desmonde, S., Oukem-Boyer, O.O.M., Nkenfou, C., Téjiokem, M., Tchendjou, P. Domkam, I., Leroy, V., Alioum, A., et al.: Estimating mother-to-child hiv transmission rates in cameroon in 2011: a computer simulation approach. BMC infectious diseases 16(1), 11 (2015)

14. Ministry of Health: Zambia Population-Based HIV Impact Assessment (ZAMPHIA) 2016: Final Report. Technical report, Ministry of Health (MOH) [Zambia] (2016). http://phia.icap.columbia.edu .

15. Central Statistical Office, Zambia and Ministry of Health, Zambia and Tropical Diseases Research Centre, University of Zambia and Macro International Inc.: Zambia Demographic and Health Survey 2007. Technical report, Macro International Inc. (2009)

16. Rodrigues, J.: Project Heart End of Project report: Tanzania, Eight years of Scaling up HIV Prevention, Care, and Treatment Services and Savings. Technical report, Elizabeth Glaser Pediatric AIDS Foundation (2012)

17. UNAIDS: UNAIDS Report on the Global AIDS Epidemic. Technical report, UNAIDS (2012)

18. FHI-360, of Health., B.S.M.: Bayelsa State-wide Rapid Health Facility Assessment. Technical report, FHI 360 and Bayelsa State Ministry of Health (2013)

19. WHO: A SHORT GUIDE: Measuring the Impact of National PMTCT Prorammes: Towards the Elimination of New HIV Infections Among Children by 2015 and Keeping Their Mothers Alive. Technical report, WHO (2012)

20. Sinunu, M.A., Schouten, E.J., Wadonda-Kabondo, N., Kajawo, E., Eliya, M., Moyo, K., Chimbwandira, F. Strunin, L., Kellerman, S.E.: Evaluating the impact of prevention of mother-to-child transmission of hiv in malawi through immunization clinic-based surveillance. PloS one 9(6) (2014)

21. Rollins, N., Little, K., Mzolo, S., Horwood, C., Newell, M.-L.: Surveillance of mother-to-child transmission prevention programmes at immunization clinics: the case for universal screening. Aids 21(10), 1341-1347 (2007)

22. Idele, P., Hayashi, C., Porth, T., Mamahit, A., Mahy, M.: Prevention of mother-to-child transmission of hiv and paediatric hiv care and treatment monitoring: from measuring process to impact and elimination of mother-to-child transmission of hiv. AIDS and behavior 21(1), 23-33 (2017)

23. Hamilton, E., Bossiky, B., Ditekemena, J., Esiru, G., Fwamba, F., Goga, A.E., Kieffer, M.P., Tsague, L.D., van de Ven, R., Wafula, R., et al.: Using the pmtct cascade to accelerate achievement of the global plan goals. Journal of acquired immune deficiency syndromes (1999) 75(1), 27 (2017)

Figure 1 Infant Sex, HIV Exposure and Vaccination Type. Panel A and B of the figure shows the distribution, in percentage, of sampled infants and HIV exposure by sex. Panel $\mathrm{C}$ shows the infant distribution by vaccination type while Panel D shows the infant HIV exposure rate disaggregated by vaccine type and sex.

Figure 2 Number of HIV positive babies by district and infant age/immunization type. The figure shows the number of HIV positive babies by immunization type tested using DNA PCR. The distribution is illustrated at both national level and disaggregated by province.

Table 1 Participation Rates

\begin{tabular}{lllllllllll}
\hline \hline Province & District & Clients & \multicolumn{3}{c}{ Contacted Consenting } & \multicolumn{4}{c}{ Refusals } \\
\hline & & & \multicolumn{2}{c}{ For Interviews } & For Blood Test & Interview & For Blood Test \\
\hline \multirow{3}{*}{ Copperbelt } & Ndola & 980 & $\mathrm{n}$ & $\%$ & $\mathrm{n}$ & $\%$ & $\mathrm{n}$ & $\%$ & $\mathrm{n}$ & $\%$ \\
& Kitwe & 987 & 970 & 98.47 & 950 & 96.94 & 15 & 1.53 & 30 & 3.06 \\
& Chingola & 939 & 930 & 99.04 & 950 & 96.25 & 17 & 1.72 & 37 & 3.75 \\
& Total & 2906 & 2865 & 98.59 & 2713 & 96.58 & 9 & 0.96 & 126 & 13.42 \\
Southern & Livingstone & 958 & 940 & 98.12 & 939 & 98.02 & 11 & 1.41 & 193 & 6.64 \\
& Choma & 1053 & 1033 & 98.10 & 1027 & 97.53 & 20 & 1.88 & 19 & 1.90 \\
& Mazabuka & 1105 & 1051 & 95.11 & 994 & 89.95 & 54 & 4.89 & 111 & 1.47 \\
& Total & 3116 & 3024 & 97.05 & 2960 & 94.99 & 92 & 2.95 & 156 & 5.01 \\
Lusaka & Lusaka & 2500 & 2400 & 96.00 & 2369 & 94.76 & 100 & 4.00 & 131 & 5.24 \\
All & & 8522 & 8289 & 97.27 & 8042 & 94.37 & 233 & 2.73 & 480 & 5.63 \\
\hline \hline
\end{tabular}


Table 2 Infant HIV exposure by primary caregiver demographic characteristics

\begin{tabular}{lccccc}
\hline & $\mathrm{N}$ & $\%$ & Infants exposed & \% exposed & P-Value \\
\hline Age of mother & & & & & \\
Less than 25 & 3,787 & 47.09 & 407 & 10.75 & $\mathrm{P}<0.001$ \\
$25-29$ & 1,962 & 24.4 & 365 & 18.60 & \\
$30+$ & 2,293 & 28.5 & 637 & 27.78 & \\
\hline Marital Status & & & & & \\
Single & 1,303 & 16.20 & 194 & 14.89 & $\mathrm{P}<0.001$ \\
Cohabiting & 55 & 0.68 & 14 & 25.45 & \\
Married & 6,561 & 81.58 & 1,158 & 17.65 & \\
Divorced & 39 & 0.48 & 11 & 28.21 & \\
Separated & 57 & 0.71 & 17 & 29.82 & \\
Widowed & 27 & 0.34 & 15 & 55.56 & \\
\hline Highest level of Education & & & & & \\
Primary & 2,300 & 28.60 & 476 & 20.70 & $\mathrm{P}<0.001$ \\
Secondary & 4,991 & 62.06 & 793 & 15.89 & \\
Higher & 457 & 5.68 & 85 & 18.60 & \\
None & 294 & 3.66 & 55 & 18.71 & \\
\hline Occupation (Employment Status) & & & & & \\
Formal employment & 625 & 7.77 & 127 & 20.32 & $\mathrm{P}<0.001$ \\
Self-employed/business & 1,899 & 23.61 & 403 & 21.22 & \\
Not employed & 5,405 & 67.21 & 865 & 16.00 & \\
School & 70 & 0.87 & 2 & 2.86 & \\
Other & 43 & 0.53 & 12 & 27.91 & \\
\hline Total & 8,042 & 100 & 1,409 & 17.52 & \\
\hline
\end{tabular}

Table 3 Overall MTCT rates by Province and age of infant/immunization type

\begin{tabular}{lllllll}
\hline & Immunization type & Penta 1 & Penta 2 & Penta 3 & Measles & Total \\
& Infant age & 6 Weeks & 10 Weeks & 14 Weeks & 9 months & \\
\hline Province & Copperbelt Province & 2.61 & 2.91 & 2.61 & 6.67 & 3.85 \\
& $\begin{array}{l}\text { Southern Province } \\
\text { Lusaka Province }\end{array}$ & 4.10 & 4.40 & 2.44 & 2.94 & 3.36 \\
& 6.85 & 1.08 & 1.05 & 6.58 & 4.15 \\
& & & & & & \\
& All & $\mathbf{4 . 7 0}$ & $\mathbf{2 . 7 9}$ & $\mathbf{2 . 1 0}$ & $\mathbf{4 . 9 9}$ & $\mathbf{3 . 7 6}$ \\
\hline
\end{tabular}


Table 4 Characteristics of HEl and HII

\begin{tabular}{|c|c|c|c|c|}
\hline \multirow{2}{*}{\multicolumn{5}{|c|}{ P-Value* }} \\
\hline & & & & \\
\hline Male & $733(55 \%)$ & $25(48.0 \%)$ & 3.41 & \multirow[t]{3}{*}{$P=0.258$} \\
\hline Female & $611(45 \%)$ & $27(51.9 \%)$ & 4.42 & \\
\hline All & $1344(100 \%)$ & $52(100 \%)$ & 3.87 & \\
\hline \multicolumn{5}{|l|}{ Age of mother } \\
\hline Less than 25 & $4401(29.8 \%)$ & $27(51.9 \%)$ & 6.73 & \multirow{4}{*}{$P=0.002$} \\
\hline $25-29$ & $3358(26.6 \%)$ & $9(17.3 \%)$ & 2.51 & \\
\hline $30+$ & $625(46.5 \%)$ & $16(30.8 \%)$ & 2.56 & \\
\hline All & $1,384(100 \%)$ & $52(100 \%)$ & 3.75 & \\
\hline \multicolumn{5}{|c|}{ Current Vaccination } \\
\hline Penta 1 & $383(27.7 \%)$ & $18(34.6 \%)$ & 4.70 & \multirow[t]{5}{*}{$\mathrm{P}=0.115$} \\
\hline Penta 2 & $287(20.7 \%)$ & $8(15.4 \%)$ & 2.79 & \\
\hline Penta 3 & $333(24.1 \%)$ & $7(13.5 \%)$ & 2.10 & \\
\hline Measles & $381(27.5 \%)$ & $19(36.5 \%)$ & 4.99 & \\
\hline All & $1,384(100 \%)$ & $52(100 \%)$ & 3.75 & \\
\hline \multicolumn{5}{|c|}{ Place of Delivery } \\
\hline Home & $111(8.0 \%)$ & $9(17.3 \%)$ & 8.11 & \multirow[t]{4}{*}{$\mathrm{P}=0.010$} \\
\hline Health Facility & $1,269(91.7 \%)$ & $42(80.8 \%)$ & 3.31 & \\
\hline Other & $4(0.3 \%)$ & $1(1.9 \%)$ & 25 & \\
\hline All & $1,384(100 \%)$ & $52(100 \%)$ & 3.75 & \\
\hline \multicolumn{5}{|c|}{ Number of ANC Visits } \\
\hline None & $5(0.4 \%)$ & $0(0.0 \%)$ & - & \multirow[t]{6}{*}{$\mathrm{P}=0.151$} \\
\hline $1-2$ & $122(8.9 \%)$ & $5(9.6 \%)$ & 4.10 & \\
\hline 3 & $426(30.9 \%)$ & $23(44.2 \%)$ & 5.40 & \\
\hline 4 & $623(45.2 \%)$ & $21(40.4 \%)$ & 3.37 & \\
\hline $5+$ & $201(14.6 \%)$ & $3(5.8 \%)$ & 1.49 & \\
\hline All & $1,377(100 \%)$ & $52(100 \%)$ & 3.78 & \\
\hline \multicolumn{5}{|c|}{ Exclusive Breast Feeding } \\
\hline Yes & $736(53.2 \%)$ & $27(51.9 \%)$ & 3.67 & \multirow[t]{3}{*}{$\mathrm{P}=0.888$} \\
\hline No & $648(46.8 \%)$ & $25(48.0 \%)$ & 3.86 & \\
\hline All & $1,384(100 \%)$ & $52(100 \%)$ & 3.75 & \\
\hline
\end{tabular}



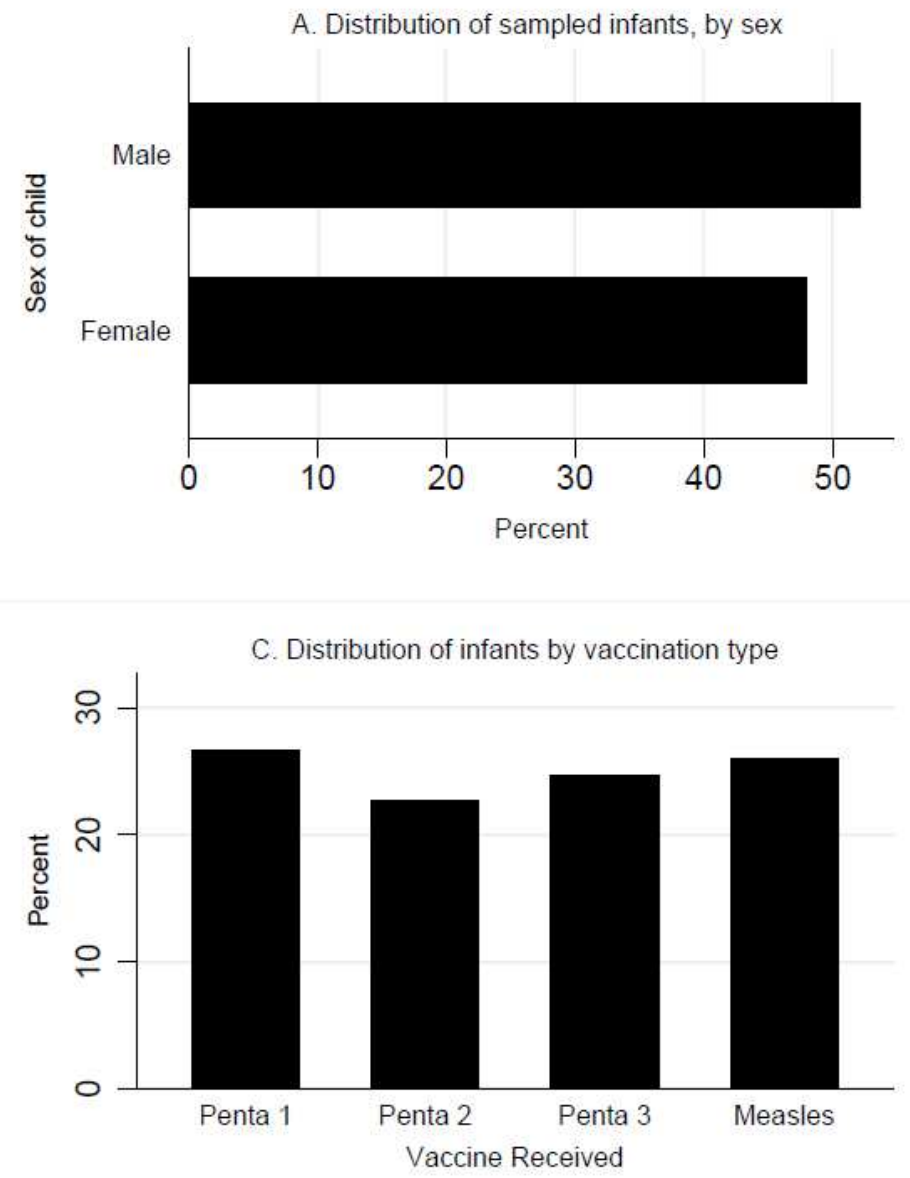

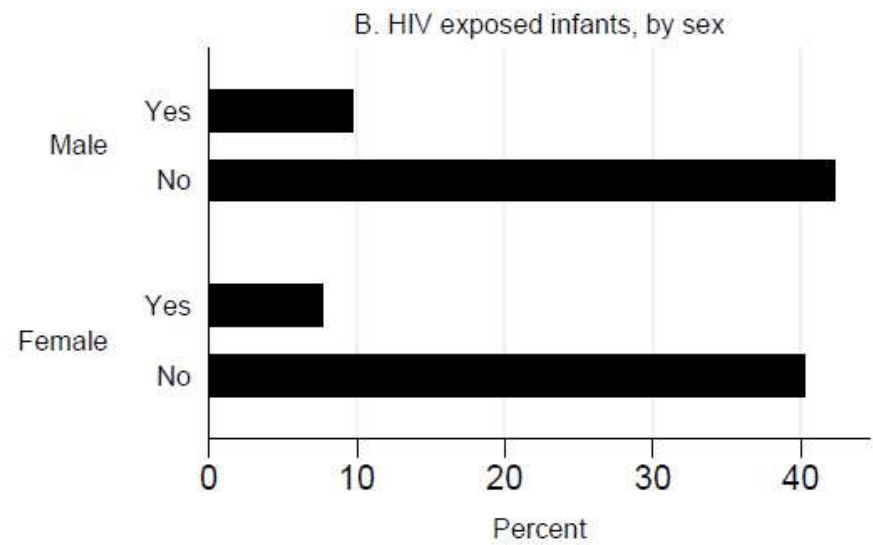

D. Infant HIV exposure rate, by vaccine type and sex

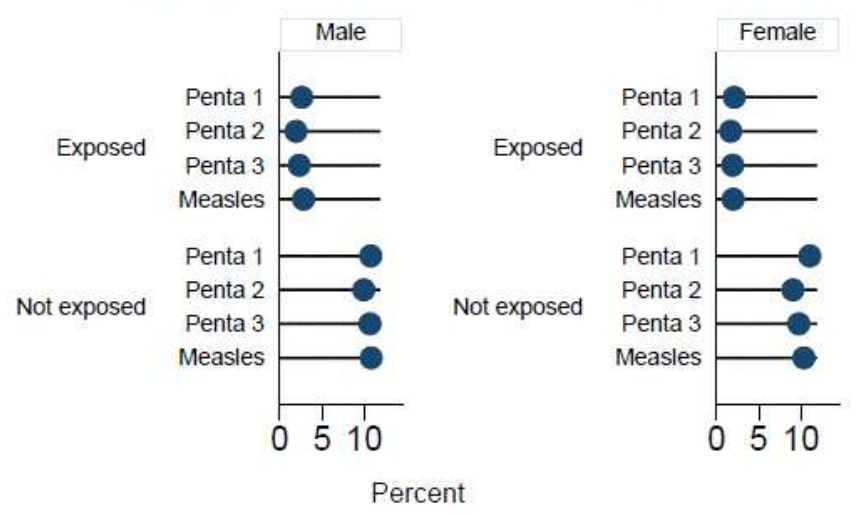

\section{Figure 1}

Infant Sex, HIV Exposure and Vaccination Type. Panel A and B of the figure shows the distribution, in percentage, of sampled infants and HIV exposure by sex. Panel $C$ shows the infant distribution by vaccination type while Panel D shows the infant HIV exposure rate disaggregated by vaccine type and sex. 

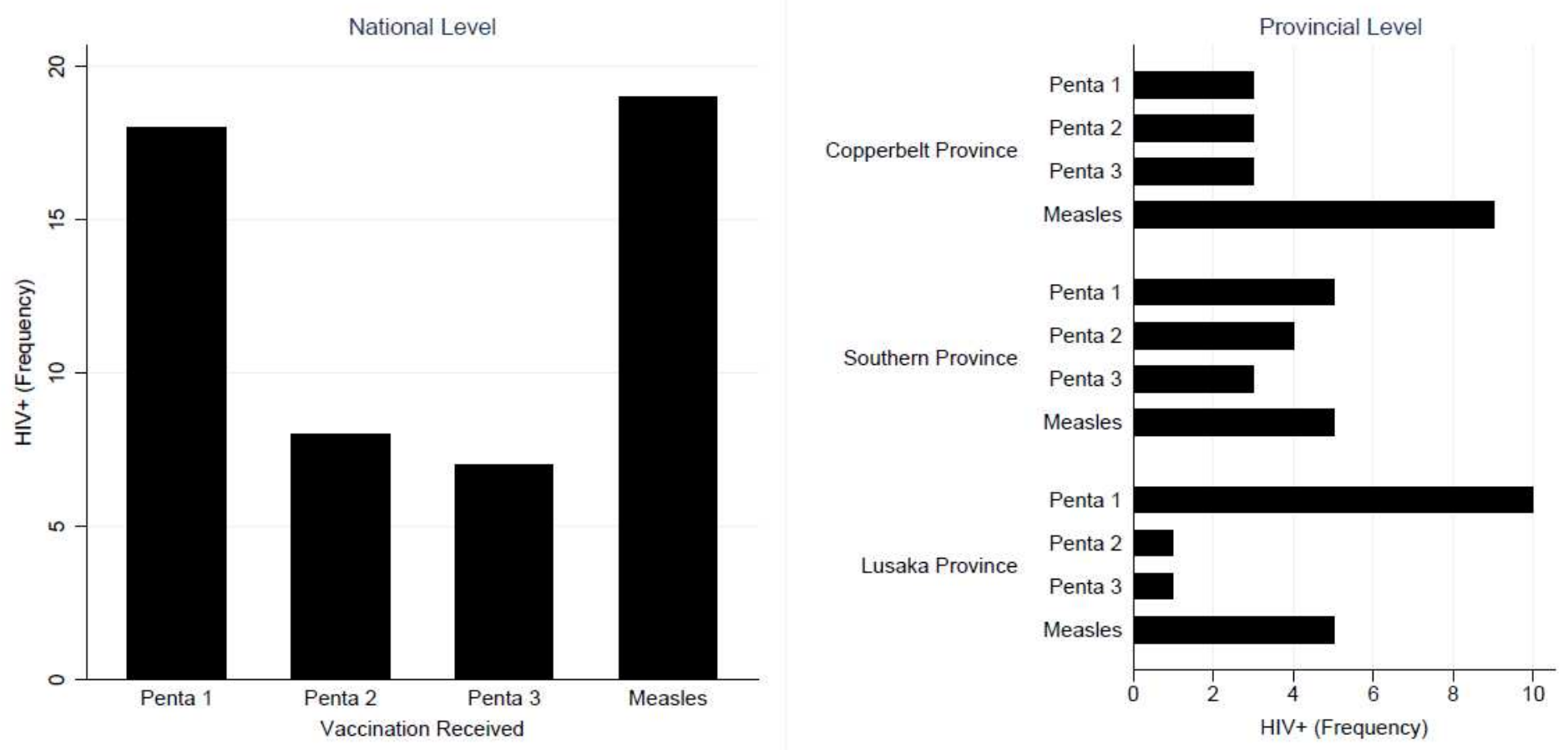

Figure 2

Number of HIV positive babies by district and infant age/immunization type. The figure shows the number of HIV positive babies by immunization type tested using DNA PCR. The distribution is illustrated at both national level and disaggregated by province. 